\title{
When the Fingers do the Talking: A Study of Group Participation with Varying Constraints to a Tabletop Interface \\ Paul Marshall ${ }^{1}$, Eva Hornecker ${ }^{2}$, Richard Morris ${ }^{1}$, Nick Sheep Dalton ${ }^{1}$ and Yvonne Rogers ${ }^{1}$ \\ 1. Pervasive Interaction Lab, Computing Dept., Open University, Milton Keynes MK7 6AA, UK \\ 2. Computer and Information Sciences, University of Strathclyde, Glasgow G1 1XH, UK \\ p.marshall@open.ac.uk
}

\begin{abstract}
A user study is presented that investigates how different configurations of input can influence equity of participation around a tabletop interface. Groups of three worked on a design task requiring negotiation in four interface conditions that varied the number (all members can act or only one) and type (touch versus mice) of input. Our findings show that a multi-touch surface increases physical interaction equity and perceptions of dominance, but does not affect levels of verbal participation. Dominant people still continue to talk the most, while quiet ones remain quiet. Qualitative analyses further revealed how other factors can affect how participants contribute to the task. The findings are discussed in terms of how the design of the physicaltechnological set-up can affect the desired form of collaboration.
\end{abstract}

\section{Introduction}

Input technologies provide the entry points into a shareable interface and the group's ongoing interaction [cf. 19, 12]. Entry points are essentially environmental structures or cues that invite or remind people to interact with the (digital) content from displays and devices. The efficacy of the entry points will depend on the loci of control and the accessibility of the mode of interaction.

In terms of the desired form of collaboration and engagement with a task we are interested in how the technological-physical set-up can encourage equitable participation among co-located groups. For example, having to stand up to write on a whiteboard in front of others can feel intimidating for some people, allowing others to dominate the interaction. However, this doesn't have to be the case. It may be possible to design interfaces that can make participation levels more rather than less equitable. This can be important for tasks such as a negotiation, where it is important that all parties feel that their views have been taken into account. In particular, we propose that the number and type of entry points to a tabletop interface can change equity of participation by providing more means of participation and making them less intimidating, so as to reduce dominance effects.

To determine if this is the case, a study was designed that compared four tabletop input conditions for a collaborative planning task. These varied in number and type of input. Groups of three carried out the task for each of the conditions. Our findings showed there to be significant differences in interactional participation but not in verbal equity. More subtle differences were evident in perceived equity. A subsequent qualitative analysis showed that the effects of changes of input condition varied depending on the group constitution, with quieter participants getting more and different chances to participate in multi-input conditions. Finally, we discuss how tabletop interfaces can be designed to constrain participation that matches the specific needs of a group of people.

\section{Background}

Equity of participation is often considered to be a desirable state, especially for tasks involving negotiation that benefit from multiple perspectives, for collaborative learning, and for tasks where it is considered important for all group members to have their say and to understand and accept the decisions being made [2]. A lack of equity is often considered undesirable, where at least one group member drops out of the discussion, i.e., does not ask questions or offer his or her opinion. In situations, where certain participants dominate the discussion, there is also the risk that relevant information is not shared, increasing the likelihood of faulty decisions [cf. 6, 8].

Benford et al [2] distinguish between approaches to the design of interfaces that either enable, encourage, and enforce collaboration. Enabling collaboration includes providing multiple entry points to allow everyone to participate at once. Encouraging collaboration can be done by providing an added benefit or more powerful functionality when working together. It is more proactive than only enabling collaboration, but not as rigid as enforcing it (e.g., strict turn-taking).

Concurrent or simultaneous interaction via interactive surfaces/tabletops has been suggested as one way of enabling more equitable participation [21]. More 'natural' 
collaboration is said to be afforded by touch input, where the interactive surface invites people to reach out and touch it with their fingers and quickly learn what to do without feeling embarrassed [23]. The degree of comfort people experience when interacting with such surfaces affects their willingness to participate. Being self-conscious can deter people from taking part, as found in studies of wall displays [e.g., 5]. People seem to prefer to interact with multi-touch tabletops, as touching a table surface is a more lightweight action than needing to stand up and go to a wall [cf. 20]. Birnholtz et al [4] have also argued that the type of input provided can have an impact on group behavior, particularly affecting individual options available by which to influence negotiation.

Morris et al [16] investigated how cooperative gestures, which require explicit coordination of group members, increases engagement. Introducing such gestures is a design approach that encourages and even enforces collaboration at certain points, which can furthermore discourage social loafing - a phenomenon that occurs where certain individuals exert less effort when working as a group than when working independently [18]. However, excessive use of cooperative gestures was found to be tedious.

Another approach to encourage collaboration is showing, in real time, visualizations of who and how much each person is talking during a meeting via a public display. For example, DiMicco [8] found that highlighting and therefore discouraging over- and under-participation changed more vocal participants' behavior: they ended up speaking less throughout the meeting. However, the effect was not symmetrical; the visualizations did not increase 'underparticipators' participation. In fact, it has the opposite effect whereby under-participators increased their participation in the control condition where there were no visualizations available. Morris et al. [16] also found that in educational settings, providing public feedback about the correctness of solutions made participants more self-conscious and discouraged participation. Private feedback, in the form of histograms that dynamically visualized the amount of speech contributions by group members, was found to increase equity. Sturm et al. [25] found feedback on speaking time and gaze via a (semi-private) peripheral display to influence both over- and under-participators towards the mean level.

Lindley and Monk [15] investigated the effects of spatial configuration around a monitor on collaboration, in terms of where people are sitting when telling stories and showing photos. They found that groups of three sitting in a semi-circle facing a monitor had higher equality and more conversational freedom (their order of turntaking was less fixed) than groups made up of two people sitting behind the person showing the photos. The number of input devices was also found to have an effect: providing several remote controls versus one when viewing the photos on a big TV resulted in more conversational turns, more overlapping talk, more freedom of turn taking and higher equality.
Single Display Groupware is an example of an enabling technology. It provides users with multiple mice and has been observed to lead to enhanced motivation, increased engagement and greater task performance for learning tasks [17]. Multiple input devices (multiple mice or tangible interaction objects) have been found to avoid a 'cursor war' [cf. 17]. This is in contrast to the single mouse situation where the partners not in control of the input device have to take effort to influence the action, often resulting in frustration and dropping out of engagement [24].

The studies investigating the effects on collaboration when using multiple versus single input devices with shared surfaces have provided mixed findings. On the one hand, parallel input has been found to decrease the opportunity for one group member to dominate while on the other it has been found to result in decreased quality of discussion, as changes are discussed less and performed individually without being negotiated. The aim of our study was to examine whether different enabling interface configurations, not only varying the number of input devices, but also the type, affect participation levels.

\section{Method}

To investigate the effects of varying the configuration of input devices on a collaborative design task when using an interactive surface, a within-subjects design was used comprising four conditions: single mouse, single touch, multi-mouse and multi-touch. All groups took part in each condition. The order of conditions was counterbalanced across groups to control for learning effects. The single and multiple mice were used as control conditions.

The main hypothesis under investigation was that the touch condition would encourage more equitable levels of participation. Providing each participant with their own means of input should also encourage greater equity of participation compared with the condition where groups have access to only one means of input. A further hypothesis was that the provision of more entry points would enable participants who speak the least to contribute to the physical design task, by carrying out more actions with the interface [cf. 19, 12].

\subsection{Task}

To maximize ecological validity we chose an openended task with no correct solution, involving the design of a seating plan for the upcoming move of a computing department at a university into a new open-plan building. All participants were going to be involved in the move. Furthermore, participants were told that their plans for seating members of the department would be provided to the management group organizing the move. Their efforts therefore could potentially have an impact on where they and others were placed in the new building. An earlier meeting of the whole department discussing criteria for seating allocation in the new building had shown it to be 
highly contentious with a number of conflicting opinions held by different members of the department.

To complete the seating allocation task, groups were provided with a floor plan depicting the physical layout on two levels, the placement of different desk configurations (e.g., 2, 4, 8 arrangements) and a set of icons representing the people who were to be moved. The two floor layouts were 'fixed' as a bird's eye 2D map; these could be grabbed, moved, and resized. The people icons could be moved around on the map by dragging them across. Additional information about people and their work connections (based on suggestions from the first departmental meeting) was provided using different colored lines. For example, people who had published together were connected with yellow lines and those involved in the same course production with green lines. People were also colorcoded, depending on whether they were administrators, faculty, technical staff or students.

The groups were asked to create their preferred seating plan by placing the people icons on to the different desks. There was no right or wrong solution but participants were encouraged to explain their criteria for where they placed people. Participants were told to take their time and that it did not matter if they did not complete the design within the available time frame.

\subsection{Participants}

39 participants volunteered from the department comprising faculty, research fellows, secretaries, administrators, technical staff and $\mathrm{PhD}$ students. They ranged in age from 22 to 65 years. 17 were female and 22 male. Participants were allocated to groups of three. This group size was chosen so as to be small enough to prevent the development of sub groups, but with an increased overhead compared to pairs required to maintain social understanding, such as the establishment of common ground [1].

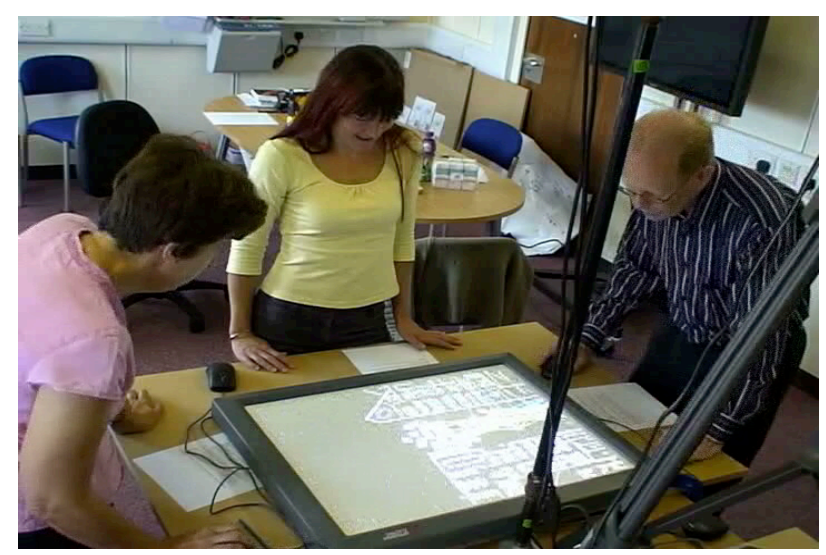

\subsection{Experimental Setup}

The standard MERL DiamondTouch surface $(65 \times 49 \mathrm{~cm})$ was used in the experiment that was accessible from three sides (see Figure 1). It can distinguish the input from multiple users [7] who either sit or stand on conductive pads. This makes it possible for participants to interact with digital content at the same time by using their fingers at the interface. In the multi-touch condition, three pads were provided on the floor for the participants to stand on. For the single touch condition one pad was provided in the central position. Participants had to switch positions to be able to take control of the surface in this condition. In the mouse condition the pads were removed and replaced with mice. In the multi-mouse condition a mouse was placed beside each participant on a larger board positioned underneath the DiamondTouch surface. In the single mouse condition one mouse was placed in front of the central participant. Again, it requires the participants to switch positions or alternatively lean over to use it.

Selecting and moving the icons in the touch condition was straight forward; involving using one finger and dragging. In the mouse condition dragging was completed using the familiar mouse dragging action. Where the two interfaces differed was for the action of zooming in and out to size and resize the floor plans. This was achieved by moving two fingers further apart or closer together in the touch condition, and using the scroll wheel in the mouse condition.

\subsection{Procedure}

13 groups of three participated in the experiment. Participants were first given a short tutorial on how to use the DiamondTouch for all four conditions. They were also given general instructions about the task. Each group worked on the same task for each of the 4 conditions, changing every 12 minutes. Overall, the task lasted for 48 minutes.

After the experiment had been completed an unstruc-

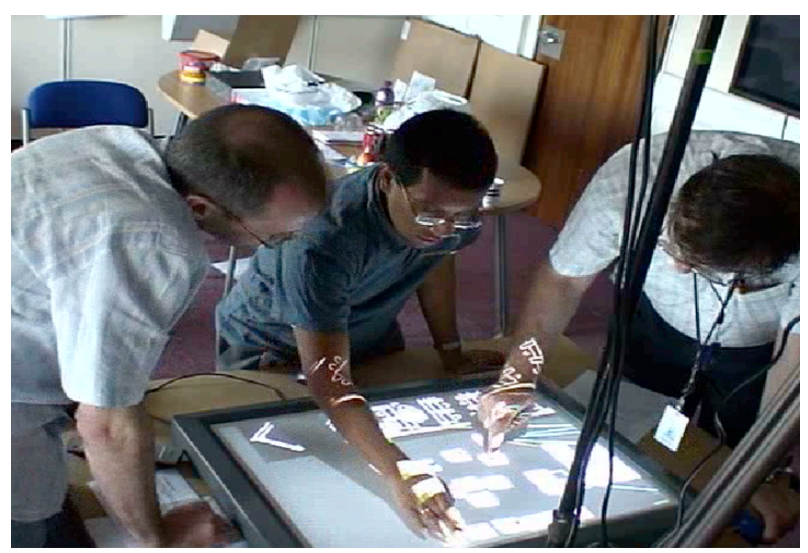

Figure 1. The study setup and interface. Left: a group working in the multiple mice condition. Right: A group working together in the multi-touch condition 
tured debriefing session was held with each group to discuss their experiences with each condition. Participants were also emailed a short questionnaire to fill out about their personal experiences of working in their group.

The sessions were videotaped with two cameras, one focusing on the interactive table and its immediate surroundings (so the mice could be observed), and the other from a wider angle, capturing the social interactions between participants.

\subsection{Indices of Participation}

Several metrics have been proposed to measure the relative contribution of individuals in a task or activity. For example, Morris et al., [16] used the standard deviation of interface actions carried out by individuals within a collaborating group as an equity metric. A standard deviation of 0 demonstrates perfect equity of participation within the group as each member has carried out the same number of actions. The larger the standard deviation, the less equitable the collaboration is. However, a disadvantage of standard deviation as an equity measure is that it varies with both group size and the total number of actions. It is therefore difficult to compare across different study designs.

The Gini Coefficient has also been used to measure the equity of contribution to groupware systems (e.g., see [9] for details of how it is calculated) and classroom dialogue [e.g., 13]. It is commonly used in economics to compare income distributions across countries, where it varies between 0 (perfect equality) and 1 (perfect inequality: 1 person has all of the income). However, this use of the Gini coefficient in its standard form is unsuitable for small numbers of participants.

We chose to use the index of inequality proposed by Hiltz et al. [11], which has been used previously in the evaluation of groupware systems. This is described below. Alternative measures are the normalized Gini coefficient as used by DiMicco et al., [8] and the measure of participation equity used by Lindlay and Monk [15].

Questionnaire data is often used to investigate perceived social presence [e.g., 10]. However, subjective perception and post-hoc reports often differ from what can be observed 'in the action' and do not provide detailed information about what people actually do [22]. We therefore considered it important to include measures of both perceived and observed equity of participation in our analysis. We chose to use a combination of three indices of equitable participation: (i) equity of interaction, (ii) verbal equity and (iii) perceived equity. The triangulation of these measures can assess more fully the multi-faceted nature of collaboration.

3.5.1 Equity of interaction. Equity of interaction was measured by coding the video data of actions and using system logs. An equity of interaction score was derived for each group of participants for each condition by first comparing system logs of interaction with the videos to determine how many system actions were carried out by each participant. The score is then calculated for each condition for each group using the formula for $I$ described below (where a low score represents greater equity of participation).

The formula I is taken from Hiltz, Turoff and Johnsons' [11] index of inequality, where $\mathrm{N}=$ size of the group; $\mathrm{E}_{\mathrm{i}}=$ the expected cumulative proportion of events if each participant contributes equally; and $\mathrm{O}_{\mathrm{i}}=$ the observed cumulative proportion of events, starting with the participant who contributed least:

$$
I=\frac{\frac{1}{N} \sum_{i=1}^{N}\left(E_{i}-O_{i}\right)}{\frac{1}{2}\left(1-\frac{1}{N}\right)}
$$

$I$ is normalized, varying between 0 (the same number of events for each participant) and 1 (all events for one of the participants). It can therefore readily be compared across studies which differ in both the number of participants and the amount of interaction.

3.5.2 Verbal equity. The same I index was used to calculate a verbal equity score, in terms of the turns taken during the conversation. Mangold Interact observational research software was used to code the number of conversational turns produced by each participant during a condition. Again, an equity score was calculated for each condition for the group using the formula for $I$.

3.5.3 Perceived equity. This was analysed using the questionnaire data. Questions asked for each of the conditions were how well each participant thought they had collaborated together; the extent to which they felt that one of the group members dominated the collaboration and the extent to which one of the group members had been left out. The participants were asked to rate each question on a 5point Likert scale. Participants were also asked to rank the four conditions in terms of preference and finally to rate on a Likert scale how much they enjoyed using each of the interface configurations.

\section{Findings}

There was much discussion with all groups suggesting a number of criteria for grouping people and where to place them in the new building. In general, it was found that the multi-touch surface supported the more equitable participation in terms of contributing to the creation of the seating plan. Below, we describe in more detail the findings for each of the three indices of participation.

\subsection{Equity of Interaction Participation}

The mean numbers of interface actions carried out by each participant are represented for each condition in Figure 2. A repeated-measures ANOVA was used to compare the 
number of interface actions produced across conditions. It revealed a main effect of type of input device, $F(1,35)=$ $8.44, \mathrm{p}<.01$, but not of number of input devices, $\mathrm{F}(1,35)=$ $0.95, p>.05$. There was also a significant interaction between the number and type of input device, $\mathrm{F}(1,35)=$ $5.07, \mathrm{p}<.05$, with the larger number of interface actions associated with using touch input being greatly increased in the multi-touch condition.

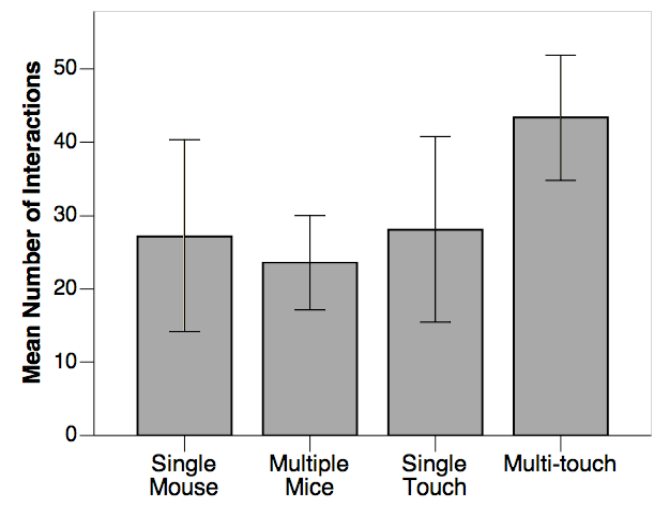

Figure 2. Mean number of physical interactions across conditions

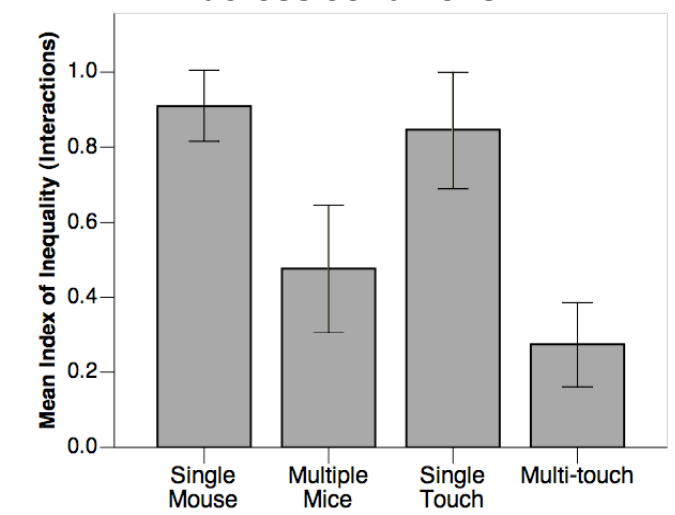

Figure 3. Mean index of inequality across conditions

The mean index of inequality for interaction participation is shown for each interface condition in Figure 3. As might be expected this was very high (almost totally unequal participation) for the single mouse condition and slightly lower for the single touch input.

A repeated-measures ANOVA detected a main effect was for both number, $\mathrm{F}(1,11)=298.81, \mathrm{p}<.001$, and type, $\mathrm{F}(1,11)=5.77, \mathrm{p}<.05$ of input device, with both multiple input devices and touch input being associated with more equitable participation (an index of inequality closer to 0 ).

\subsection{Equity of Verbal Participation}

The number of conversational turns produced by each participant was compared across the four conditions using a repeated-measures ANOVA. There was no significant effect of either number, $\mathrm{F}(1,29)=2.10, \mathrm{p}>.05$, or type, $\mathrm{F}(1$,
$29)=0.36, p>.05$ of input device on the number of conversational turns.

An index of inequality was calculated for each group in each of the four conditions. A comparison with a repeatedmeasures ANOVA found no effect of either number, $\mathrm{F}(1$, $10)=0.002, p>.05$, or type, $F(1,10)=1.61, p>.05$ of input device on the equity of verbal participation. Therefore, it would appear that more equitable verbal collaboration is not encouraged by simply providing users with more entry points to a collaborative surface or by enabling touch input.

\subsection{Perceived Equity}

Questionnaire responses indicated that the participants perceived there to be no effect of either type, $F(1,28)=$ $2.07, \mathrm{p}>$. 05, or number, $\mathrm{F}(1,28)=0.39, \mathrm{p}>.05$, of input device in supporting working together effectively as a group. However, participants were significantly more likely to report that one member of the group was able to dominate the task when using mice compared with using touch input, $\mathrm{F}(1,28)=10.0, \mathrm{p}<.05$. No effect of number of devices was found, $\mathrm{F}(1,28)=0.01, \mathrm{p}>.05$. Therefore, participants suggested that one participant was able to dominate the task in the mouse conditions regardless of whether one or three mice were available for use. Mean ratings for perceived dominance are represented in Figure 4.

Similarly, participants reported that one person was more likely to contribute little to the task in the two mice conditions than when using touch input, $\mathrm{F}(1,24)=4.55, \mathrm{p}<$ .05 (see Figure 5).

A significant main effect was also found for the number of inputs, $\mathrm{F}(1,24)=6.00, \mathrm{p}<.05$, with one participant less likely to be left out of the design task when multiple inputs were being used than when only a single input device was available.

Therefore, more entry points were perceived as making it possible for more people in the group to contribute. Furthermore, the touch conditions were perceived as raising the levels of contribution of group members who otherwise would contribute the least.

An analysis of participants' interface preferences (see Figure 6) revealed a significant effect of interface condition, $\chi^{2}(3)=18.57, \mathrm{p}<.001$. Post-hoc Wilcoxon tests revealed that both the single touch, $\mathrm{T}=67.5, \mathrm{p}<.001$, and the multi-touch input conditions, $\mathrm{T}=133.0, \mathrm{p}<.05$ were preferred to single mice. The multiple mice were the least preferred input configuration, but the difference between multiple and single mice was found to not be significant, $\mathrm{T}$ $=153, \mathrm{p}>.05$. There was also no significant difference between preferences for the two touch input conditions, $\mathrm{T}=$ $176.0, p>.05$. Therefore, participants expressed a preference for touch input, but none for more input devices. Similarly, a main effect was found for the type of input device on participants' rated level of enjoyment, $\mathrm{F}(1,30)=14.69, \mathrm{p}<$ .001 , with touch input being enjoyed more. 


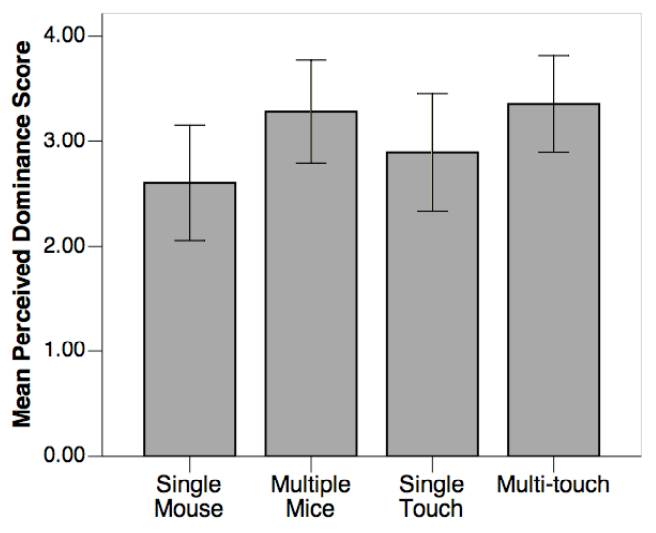

Figure 4. Perceived Dominance by one person in each condition $(1=$ agree very much; $5=$ not at all $)$

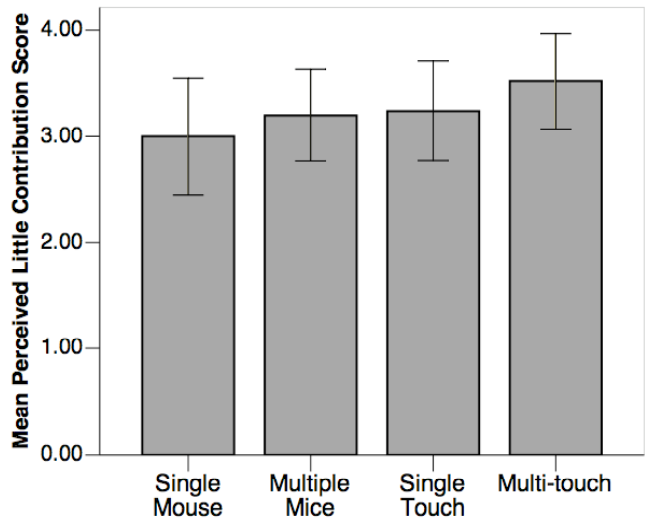

Figure 5. Perceived little contribution by one person $(1=$ agree very much; $5=$ not at all)

\subsection{Discussion of Quantitative Findings}

Taken together, the three measures have revealed that influencing the way groups can interact with a shared surface has an effect on the way they contribute, physically to the task. The implication is that greater interaction equity can be encouraged by simply providing more input devices and touch input. Touch input has also been found to increase the quantity of interface actions for all participants by reducing the interaction overhead.

Findings for interaction equity were however not echoed in the findings for verbal participation or for overall perception of effective group working, where no differences were found across conditions. This would appear to suggest that participants' perceptions of effective group working are influenced primarily by the nature of the verbal discussion rather than equity in interacting with the interface.

Yet, having multiple input devices at hand was perceived to decrease the ability of an individual to dominate the discussion. Touch input was perceived making it less likely that a single person would be left out of the task. This would seem to suggest that while perceptions of equal collaboration are largely related to talk, more subtle effects related to perceived dominance or under-participation are influenced by what people do as well as what they say.

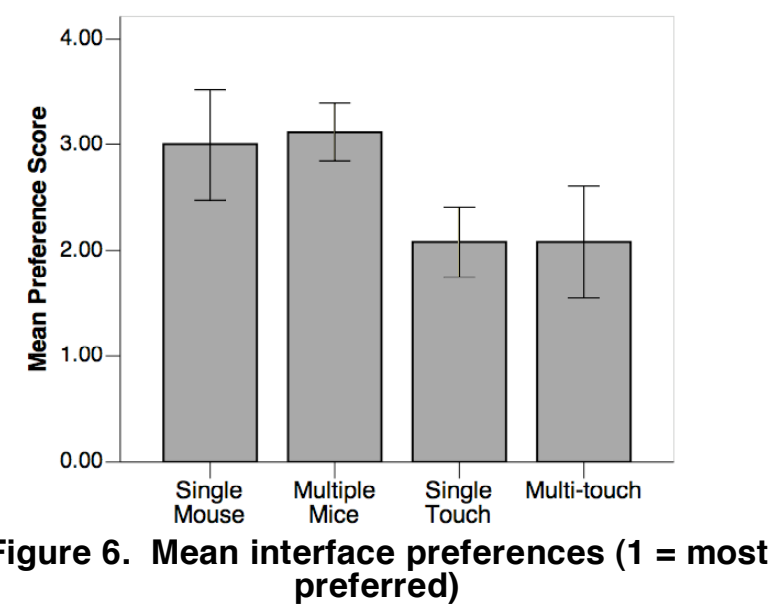

To further investigate these findings we subsequently returned to the videos to analyze aspects of individual group dynamics. A number of groups were found to respond to the changes in interface conditions in different ways, suggesting that individual differences may be an important factor when designing to encourage equitable participation.

\subsection{Qualitative Analysis}

The effects of input condition were found to affect groups differently depending upon the general group dynamics. We describe these in terms of how well each knew each other, status and individual differences.

4.5.1. Homogenous groups in terms of talk levels. For groups of reasonably well-matched participants (all quite quiet or all talkative) we observed diminished effects of number and type of input devices - people adapted their interaction patterns to suit the overall working style.

For example, one group comprised three individuals who did not know each other very well and were all fairly quiet. In this case, equity of verbal collaboration was consistently high and evenly distributed across conditions. Conversation was characterized by little overlap in conversational turns: each participant tended to wait for the others to finish their point before expressing their own opinions. A similar situation resulted in a group where the three participants working together knew each other very well and shared a similar status in the departmental hierarchy. Their conversation was much more animated, with frequent overlaps between conversational turns, more talk in total, and uniformly high verbal participation across conditions.

4.5.2. Status effects. A different situation resulted in groups with significant differences in status and knowledge on the task. Participants with a higher status (e.g. managers or professors), who tended to know the departmental structure well, often dominated, while lower status participants, such as $\mathrm{PhD}$ students, tended to be more passive. In this situation, multiple input, and in particular multi-touch increased the involvement of people that in the other conditions had only been marginally involved. 
In one group consisting of two professors and a $\mathrm{PhD}$ student, the two higher status individuals mainly talked with each other, although did attempt to involve the student. With the single input devices, one of the professors took control and the $\mathrm{PhD}$ student was unable to contribute greatly to the design of the seating plan. With multiple access points however, the student was able to lean in and make changes of his own. This also led to a greater involvement in the discussion.

In another group of similar constellation, two higherstatus people tended to discuss the task at an abstract level. This led to the student taking on a rather active role in manual contributions across conditions, even though she did not talk much. Her interactions at times were a direct reaction to the ongoing talk, and at times were of her own volition, pragmatically getting the task done during discussions of higher-level aspects. In this case, there was little effect of using multiple input devices on verbal participation.

A third group had a different status relationship, where one person (Bob) was the supervisor of another (Ken), a student like the third participant (George). Here, in the single input conditions, a dyadic interaction evolved, with Bob instructing and discussing with Ken who controlled interface manipulations. George was largely excluded from the discussion. In the multiple input conditions however, George was able to contribute verbally and physically and verbal equity of participation of the group doubled.

4.5.3. Individual differences. In another group the status differences were less pronounced, but different personalities led to differential levels of participation. Here, one of the participants was quiet, resulting in two of the highest inequality scores $(I \approx 0.35)$ in the single user conditions. In the multiple input device conditions however, this person was much more active, also resulting in a greatly increased level of verbal participation ( $I \approx 0.20)$.

\section{Discussion}

Our study has shown how different entry points to a tabletop interface can influence collaboration in a shared design task involving negotiation. Our focus was on whether more equitable collaboration could be enabled through allowing all group members to easily participate using a multi-touch tabletop. We compared this with a control condition of single interaction and mouse input. A main finding was that equity of interaction with the tabletop interface was increased by the provision of both touch and multiple entry points. However, there was no effect on equity of verbal participation. Participant's perceptions of equitable collaboration were subtler, with more entry points perceived as increasing opportunities for people in the group to contribute and the touch input seen as raising the levels of contribution of group members who otherwise would contribute the least.
It remains unclear whether these findings will extend to different kinds of tasks and interface configurations. It seems plausible, for example, that the increased cost of maintaining awareness with a much larger touch surface might lead to a greater level of verbal equity through uniformly increased verbal shadowing.

A further question that has not been addressed here is whether simply comparing the quantity of dialogue is an adequate test of verbal equity. It seems at least plausible that a participant might say quite little, but still have a significant influence on the path of the conversation. We plan to develop our analysis to include the content of dialogue in future work.

Our findings suggest that designing tabletop surfaces to enable collaboration may be sufficient for co-located groups working on design tasks where interaction with digital content is central. Other researchers have proposed a more 'heavyweight' approach to encourage more equitable collaboration through the provision of awareness visualizations representing the verbal [8] or interactional [16] levels of contribution of different group members or through manipulating the feedback modality or privacy [16]. This method has been found to reduce the influence of the more dominant group members [8] - since presumably their contribution is explicit for all to see making all more aware of their dominance. However, it has been less effective in encouraging participation in quieter participants, where it may actually have the opposite effect.

Another way of encouraging (or even enforcing) collaboration has been to use cooperative gestures [16], which provide magnified or additional commands when groups work together. This approach trades efficiency of interaction against any collaborative benefits such as a reduction of conflicting actions. The most heavyweight approach that has been suggested is to enforce collaboration by building constraints into a system. This has been shown to be effective in specific situations where providing scaffolding has been identified as important, such as encouraging children on the Autistic spectrum to work together [16] and supporting learning [cf. 14].

Hence, a one-size-fits-all approach is inappropriate when designing technologies to facilitate more equitable verbal discussions during group work [see also 4, 8]. Further research is needed to determine which user groups and individual types may benefit from having more explicit constraints and/or visual feedback at the tabletop interface that can enforce cooperation and which in turn may modulate verbal participation levels. If the goal is simply to encourage a more equal contribution in the interactive task, then enabling technologies alone may be suitable. If the goal is to decrease verbal participation by dominant individuals, then awareness visualizations representing their (over)contribution may be appropriate. It still remains unclear how to encourage verbal participation from otherwise reticent collaborators [8]; awareness visualizations could have a negative effect. We suggest that it may be enough that they are able to participate in the interaction 
without having to verbally contribute. Being able to interact with a tabletop may provide a valuable side channel for less verbose or self-confident participants and may allow them to take part in a way they feel comfortable with [cf. 19].

\section{Conclusion}

Our study has shown that tabletop interfaces can be designed to enable more equitable participation without necessarily having to be encouraged, or constrained through enforced turn taking. We have shown that this kind of enabling interface can lead to fingers rather than voices doing the talking: interactive participation is more equal with touch input and multiple entry points than with mice or single input, but verbal participation is not. Our research has also begun to uncover some relationships between tabletop affordances and group composition. Whether it is possible to engender different ways of talking in co-located groups through the provision of different forms of tabletop interface remains to be seen.

\section{Acknowledgements}

We thank those who volunteered to take part in the study and our reviewers for their helpful comments. This research was supported by an OU research fellowship, the EPSRC ShareIT project EP/F017324/1 and the German DFG grant Ho3901/1-1.

\section{REFERENCES}

[1] Anderson, A.H. Achieving Understanding in face-to-face and video-mediated multiparty interactions. Discourse Processes 41,(3), 2006, 251-287

[2] Arias, E., H. Eden, G. Fischer, A. Gorman, E. Scharff, Transcending the Individual Human Mind. Creating Shared Understanding through Collaborative Design. ACM Transactions on Computer-Human-Interaction (ToCHI) 7 (1) 2000, 84-113.

[3] Benford, S. et al., Designing storytelling technologies to encouraging collaboration between young children. Proc. of CHI'00. ACM 2000. 556-563

[4] Birnholtz, J.P., T. Grossman, C. Mak, R. Balakrishnan, An exploratory study of input configuration and group process in a negotiation task using a large display. Proc. of CHI'07. ACM 2007. 91-100

[5] Brignull, H., Y. Rogers, Enticing people to interact with large public displays in public spaces. Proc. of INTERACT'03. 2003, 1724

[6] Brown, R., Group Polarization, in Brown, R. (ed.) Social Psychology. Free Press, New York 1986. 200-248.

[7] Dietz, P.H., D.L. Leigh, DiamondTouch: A Multi-User Touch Technology. Proc. of UIST'01. ACM 2001. 219-226

[8] DiMicco, J.M., A. Pandolfo, W. Bender, Influencing group participation with a shared display. Proc. of $C S C W^{\prime} 04$. ACM 2004, 614-623
[9] Fitze, M. (2006) Discourse and Participation in ESL Face-toFace and Written Electronic Conferences. Language Learning \& Technology, Vol. 10, No. 1.67-86

[10] Harms, C., Biocca, A.F. (2004) Internal consistency and reliability of the networked minds social presence measure. In Alcanizm, Rey (Eds.), Seventh Annual International Workshop: Presence 2004.

[11] Hiltz, S.R., M. Turoff, K. Johnson, Experiments in Group Decision Making, 3: Disinhibition, Deindividuation, and Group Process in Pen Name and Real Name Computer Conferences. Journal of Decision Support Systems, (5), 1989, 217-232.

[12] Hornecker, E., P. Marshall, Y. Rogers, Entry and Access How Shareability Comes About. Proc. of DPPI'07. ACM 2007, $328-342$

[13] Kelly, S. (2007) Classroom discourse and the distribution of student engagement. Social Psychology Education 10:331-352

[14] Kerawalla, L., Pearce, D., Yuill, N., Luckin, R., and Harris, A. 2008. "I'm keeping those there, are you?" The role of a new user interface paradigm - Separate Control of Shared Space (SCOSS) - in the collaborative decision-making process. Comput. Educ. 50, 1 (Jan. 2008), 193-206.

[15] Lindley, S., A. Monk, Social enjoyment with electronic photo displays: awareness and control. Submitted to the International Journal of Human-Computer Studies

[16] Morris, M. R., A. Cassanego, A. Paepcke, T. Winograd, A.M. Piper, A. Huang, Mediating Group Dynamics through Tabletop Interface Design. IEEE Computer Graphics and Applications (Sept/Oct) 2006, 65-73

[17] Pawar, U.S., J. Pal, R. Gupta, K. Toyama, Multiple Mice for Retention Tasks in Disadvantaged Schools. Proc. of CHI'07. ACM 2007. 1581-1590

[18] Pennington, D.C. The Social Psychology of Behaviour in Small Groups. Psychology Press Ltd/Taylor \& Francis, 2002.

[19] Rogers, Y., Y. Lim, W.R. Hazlewood, P. Marshall, (in press) Equal Opportunities: Do Shareable Interfaces Promote More Group Participation Than Single User Displays. Accepted for publication. Human Computer Interaction

[20] Rogers, Y., S. Lindley, Collaborating around vertical and horizontal displays: which way is best? Interacting With Computers, 16. 2004, 1133-1152

[21] Scott, S.D., K.D. Grant, R.L. Mandryk, System guidelines for co-located, collaborative Work on a Tabletop Display. Proc. of ECSCW'03. 2003

[22] Sharp, H., Rogers, Y., Preece, J. (2007) Interaction Design Beyond Human-Computer Interaction. 2nd edition, John Wiley, Chichester

[23] Shen, C., Everitt, K.M., Ryall, K. (2003) UbiTable: Impromptu Face-to-Face Collaboration on Horizontal Interactive Surfaces," Proc. of UbiComp'03, 281-288.

[24] Stewart, J., E. Rayborn, B. Bederson, A. Druin, When Two Hands are Better Than One. Proc. of CHI'98 extended abstracts, ACM, N.Y. 1998, 287-288

[25] Sturm, J., Houben-van Herwijnen, O., Eyck, A., Terken, J. (2007) Influencing social dynamics in meetings through a peripheral display. Proc. of ICM'07. ACM. 263-270 